\title{
INVOCACIONES Y USOS INADECUADOS DE LA CIENCIA EN LA PUBLICIDAD
}

\author{
CAMPANARIO, JUAN MIGUEL, ${ }^{1}$ MOYA, AIDA ${ }^{2}$ y OTERO, JOSÉ C. ${ }^{3}$ \\ Grupo de Investigación sobre el Aprendizaje de las Ciencias. Departamento de Física. \\ Universidad de Alcalá. 28871 Alcalá de Henares. Madrid \\ 1 fscampanario@alcala.es \\ 2 fsegp@alcala.es \\ 3 fsotero@alcala.es
}

\begin{abstract}
SUMMARY
The use of science in advertising as a source of authority to back claims concerning products is studied in this article. We also examine inappropiate uses of scientific knowledge in advertising. The analyzed examples present an image of science as an activity always in progress that is able to guarantee the quality of products. Among the wrong uses of science we found the use of supossed scientific concepts, the use of misleading or difficult to understand argumentations, or the misuse of scientific concepts. These uses are in conflict with goals of science education that emphasize understanding of scientific knowledge, and its correct use.
\end{abstract}

\section{INTRODUCCIÓN}

La publicidad es uno de los fenómenos característicos de nuestro tiempo y es uno de los elementos que sirve para moldear la opinión de los ciudadanos y para crear hábitos de comportamiento. El discurso publicitario contribuye a configurar modelos, estereotipos e incluso objetivos y planteamientos vitales. El economista John Kenneth Galbraith considera que la publicidad es un componente clave del sistema socioeconómico actual que, con su enorme poder e influencia, contribuye a mantener en marcha todo el sistema productivo (Galbraith, 1992).

La inversión en publicidad constituye, con frecuencia, una parte considerable de los gastos que deben abordar las empresas y firmas comerciales. Por ejemplo, en España la inversión publicitaria en medios convencionales (televisión, diarios, suplementos y dominicales, revistas, radio, cine y publicidad exterior) ascendió en 1996 a 608.686 millones de pesetas y representó el
$48,6 \%$ del total del gasto publicitario en España en dicho año (Infoadex, 1997). Por su relación con el contenido de este trabajo, es interesante destacar que los diarios ocupan el segundo lugar en el ranking, después de la televisión, con 191.486 millones de pesetas (el 31,5\% del total de la inversión publicitaria en medios convencionales), mientras las revistas, incluidas las revistas técnicas, constituyen el tercer medio convencional por inversión publicitaria con 78.563 millones de pesetas (el 12,9\% del total). Suplementos y dominicales recibieron 16.098 millones de pesetas (el 2,6\% del total de los medios convencionales de publicidad).

El estudio y análisis de la publicidad y el marketing ha dado origen a diversas líneas de investigación que abordan estos temas desde distintas perspectivas. Existen numerosos libros y revistas (por ejemplo, Journal of Consumer Research, European Journal of Marketing o 
Journal of Advertising) que tratan temas diversos, desde la eficacia de la publicidad y su efecto en los consumidores, a los aspectos éticos y económicos. Otros autores han analizado los aspectos jurídicos de la publicidad (Morgan y Stoltman, 1997), el uso del lenguaje en la publicidad (McQuarrie y Mick, 1996; Cook, 1992) o las argumentaciones que aparecen en los anuncios (van Eemeren, Grootendorst, Jackson y Jacobs, 1997).

Con frecuencia la ciencia aparece relacionada con la publicidad. La forma más frecuente de asociación es aquélla en la que la ciencia constituye una fuente de autoridad que garantiza la calidad de los productos que se anuncian. Este tipo de recurso a la ciencia no es algo nuevo. Así, por ejemplo, los anuncios de cosméticos en la prensa escrita de los años veinte, reflejan claramente la idea muy extendida en aquella época de que la ciencia sería capaz de curarlo prácticamente todo, incluyendo el envejecimiento (Raymond, 1988). Una idea similar a la anterior es la que se transmite con frecuencia actualmente. Según el estereotipo de la ciencia como garantía de certeza, común en la prensa escrita, los científicos son «quienes resuelven los problemas, la última fuente de verdad» (Nelkin, 1991, p. 134). Según un estudio reciente, la confianza y el interés del público en los Estados Unidos por la ciencia ha crecido en los últimos años, aunque esta confianza no va acompañada de una comprensión básica de la misma (Macilwain, 1998).

El conocimiento científico ha pasado a ser el paradigma de conocimiento riguroso, fiable y exacto. El conocimiento científico sirve como modelo para otras disciplinas que pugnan por añadir el adjetivo «científico» a sus métodos y conclusiones. Los estudiantes universitarios, por ejemplo, mantienen creencias exageradas sobre la ciencia y el conocimiento científico: conciben el conocimiento científico como superior a otras clases de conocimiento. Incluso, en casos extremos, tales como al encontrar contradicciones explícitas en textos supuestamente científicos, los alumnos mantienen a veces actitudes reverenciales absteniéndose de manifestar su falta de comprensión (Otero, 1987; Otero y Campanario, 1990). Esta superioridad se derivaría del método que se utiliza en la obtención del conocimiento científico (Porlán, 1994) y va asociada, a veces, a una concepción de la ciencia como una actividad misteriosa que se sitúa «fuera, y por encima, de la capacidad de comprensión humana normal, y que queda, por tanto, fuera del alcance de toda crítica seria» (Nelkin, 1991, p. 134).

El carácter provisional y tentativo de las teorías científicas no siempre se hace explícito a los alumnos. A veces, el conocimiento científico se presenta como un conocimiento acabado y fuera de toda duda o no se insiste suficientemente en la indagación como componente clave del conocimiento científico. Por ejemplo, un análisis lingüístico del contenido de libros americanos de biología de enseñanza secundaria demostró que el número de referencias a la ciencia como indagación disminuyó a partir de 1977 (Eltinge y Roberts, 1993). Los libros de texto de ciencias de enseñanza secundaria suelen contener afirmaciones que retratan la ciencia como una actividad que contiene verdades fijas e inalte- rables (Meichtry, 1993). Muchos profesores de ciencias mantienen también visiones inadecuadas sobre la naturaleza de la ciencia (Lederman, 1992) y probablemente las transmiten a sus alumnos explícita o implícitamente. Según estas visiones inadecuadas, la ciencia estaría formada por proposiciones probadas fuera de toda duda. Sin embargo, esta visión justificacionista de la ciencia ha sido abandonada por científicos y filósofos de la ciencia. Como afirma Lakatos, «actualmente son muy pocos los filósofos o los científicos que todavía piensan que el conocimiento científico es, o puede ser, conocimiento demostrado» (1975, p. 240).

Una parte de la responsabilidad de estas visiones inadecuadas puede que recaiga también en la forma en que se presenta la ciencia y la tecnología en los medios de comunicación. Los periódicos y revistas populares suelen referirse a la ciencia y a la actividad de los científicos como algo aparte de las actividades humanas normales (Nelkin, 1999, p. 31). Por ejemplo, en un análisis del contenido de la revista Time, relativo a noticias sobre ordenadores y tecnologías relacionadas, Stahl descubrió que, en el $36 \%$ de los casos, se utilizaba explícitamente un lenguaje relacionado con la magia o la religión (Stahl, 1995). Así, términos comomago de la informática, artes secretas, paraíso electrónico, nuevos conversos o milagroso forman parte del repertorio de expresiones asociadas comúnmente a las nuevas tecnologías y a quienes las venden o utilizan. Los propios científicos, a veces, cuando hablan a los periodistas insisten en la complejidad de su trabajo y lo presentan como algo difícil de explicar y de entender (Nelkin, 1990, p. 31). Además, los aspectos novedosos de la ciencia siempre llaman más la atención de los medios de comunicación. Así, los científicos son presentados habitualmente como «guerreros que combaten la enfermedad, conquistan las fuerzas de la naturaleza [...] están comprometidos con una revolución, bien se trate de una revolución biotecnológica o una era revolucionaria [...] o un avance de dimensión revolucionaria» (Nelkin, 1991, p. 130).

El recurso a la ciencia en la publicidad no es ajeno al papel que ésta desempeña en nuestra sociedad. Si tuviésemos que comparar la cultura actual con otras anteriores, uno de los rasgos diferenciales sería probablemente el papel que en ella desempeñan la ciencia y la tecnología. Además, la mayor parte de los bienes y productos que consumimos son elaborados siguiendo complejos procesos basados en investigaciones científicas o en desarrollos tecnológicos. En palabras de Yearley, «la ciencia nos proporciona ordenadores y aviones, rayos X y pilas de larga duración. La ciencia está tan estrechamente identificada con los productos innovadores y exitosos que las empresas intentan llevar el agua al molino en sus anuncios» (Yearley, 1993-94, p. 59). No resulta raro, pues, que el discurso publicitario recurra a la ciencia en la fase de promoción de los bienes y productos, dado que, en general, la ciencia ha acompañado a muchos de estos bienes y productos desde la fase de proyecto.

A pesar de las importantes relaciones entre ciencia y publicidad, no conocemos trabajos que aborden su estu- 


\section{INVESTIGACIÓN DIDÁCTICA}

dio. El objetivo de este artículo es analizar las invocaciones y usos inadecuados de la ciencia en la publicidad. En primer lugar se analiza el uso publicitario de la ciencia como fuente de autoridad que respalda las virtudes de los productos y servicios anunciados. En segundo lugar, se analiza la frecuente asociación de este uso de la ciencia con una presentación errónea de los contenidos científicos y con razonamientos incorrectos mediante los que se llega a conclusiones de interés para el anunciante.

\section{MÉTODO}

En este trabajo se presentan y analizan ejemplos tomados de publicidad escrita. Los anuncios proceden de periódicos de difusión nacional (El País, El Mundo...), revistas generales, no dirigidas a especialistas (Hola, Cambio 16...) y suplementos dominicales de periódicos de difusión nacional. Los anuncios utilizados no se han seleccionado de manera sistemática o aleatoria y, por tanto, no se puede considerar que sean una muestra representativa. Los ejemplos que se analizan y discuten en este trabajo constituyen una muestra de conveniencia. Un estudio con mayor validez externa quedaría para trabajos futuros. En este futuro trabajo se podría cuantificar, por ejemplo, la frecuencia de anuncios inadecuados desde el punto de vista científico.

El origen de la colección está en la necesidad de disponer de un repertorio de anuncios que puedan ser utilizados en tareas docentes como ilustración de lo que no es el contenido ni los métodos de la ciencia. De manera similar, otros autores han sugerido el uso de noticias periodísticas como un recurso en la enseñanza de las ciencias (Manrique del Campo, 1995). A medida que se identificaban nuevos casos, pareció evidente que éstos podían clasificarse de acuerdo con algunas categorías

Tabla I

Ejemplos de anuncios en los que se hacen invocaciones a la ciencia o a la tecnología.

\begin{tabular}{|c|c|}
\hline $\begin{array}{l}\text { TIPO DE } \\
\text { PRODUCTO }\end{array}$ & CONTENIDO DEL ANUNCIO \\
\hline Detergente & La ciencia al servicio de la limpieza. \\
\hline Servicios bancarios & Fuerte en nuevas tecnologías. \\
\hline Neumáticos & La ciencia de la seguridad. \\
\hline Cosméticos & Nunca la ciencia ha hecho tanto por la belleza. \\
\hline Crema hidratante & $\begin{array}{l}\text { Resultado obtenido después de diez años de investigación en colaboración con el CNRS (Centro Nacional de } \\
\text { Investigación Científica de Francia). }\end{array}$ \\
\hline Crema anticelulítica & [...] te ofrece la solución científica y eficaz contra la celulitis. \\
\hline Almohada cervical & $\begin{array}{l}\text { La mariposa cervical es la única almohada desarrollada científicamente bajo control médico y recomendada por } \\
\text { especialistas para el tratamiento y prevención del dolor. }\end{array}$ \\
\hline Zapatos & $\begin{array}{l}\text { Los [nombre del producto] son el resultado de cuatro años de investigación del Instituto de Biomecánica de } \\
\text { Valencia. }\end{array}$ \\
\hline Crema hidratante & $\begin{array}{l}\text { Como resultado de la investigación cosmetológica más avanzada con los más recientes descubrimientos } \\
\text { biológicos y vegetales. }\end{array}$ \\
\hline Automóviles & A la vanguardia de la técnica. \\
\hline Teléfono móvil & Nueva dimensión tecnológica de baterías. \\
\hline Lavavajillas & Su tecnología es tan avanzada que no se cuestiona. \\
\hline Crema & El origen de [nombre del producto] se fundamenta en un descubrimiento científico revolucionario. \\
\hline Productos cosméticos & El complejo [nombre del producto] sintetiza los últimos descubrimientos científicos en la biotecnología. \\
\hline Crema hidratante & Eficacia científicamente probada. \\
\hline Crema antiarrugas & Un triunfo antiarrugas demostrado por la investigación dermocosmética. \\
\hline Cereales & Por todo ello, el Instituto Español de la Nutrición, avala la calidad nutricional de [...] \\
\hline
\end{tabular}


generales. Estas categorías se determinaron de manera inductiva y permiten clasificar la colección de anuncios que aquí se presenta. No conocemos precedentes en la literatura acerca de este tipo de trabajos, por ello, la clasificación que aquí se propone es tentativa y sujeta a futuras modificaciones. Los criterios que permiten clasificar los ejemplos en las diversas categorías se detallan más adelante.

\section{RESULTADOS}

Hemos clasificado los anuncios en dos amplias categorías: invocación a la ciencia como fuente de autoridad y usos inadecuados del lenguaje o del conocimiento científico. La primera categoría recoge aquellos ejemplos en los que la ciencia, con su supuesta autoridad, sirve para respaldar explícita o implícitamente un producto. La segunda categoría recoge aquellos ejemplos en los que el lenguaje o el conocimiento científico se utilizan de forma inadecuada. Estos usos inadecuados se clasifican, a su vez, en distintas categorías, dependiendo del error en el que se incurre o de la orientación general del anuncio que se analiza.

Dado el carácter del estudio que se presenta en este trabajo, no se intenta hacer una evaluación cuantitativa del número de anuncios que pueden clasificarse de acuerdo con cada una de las categorías. Como se ha indicado más arriba, ello exigiría seguir otro enfoque metodológico y tener en cuenta también los anuncios «correctos» desde el punto de vista científico.

\section{Invocaciones a la ciencia como fuente de autoridad}

En la tabla I se recogen anuncios en los cuales se invoca a la ciencia, a la tecnología o a la investigación científica como garantías de calidad o eficacia de productos o servicios, o como fuentes de autoridad para las afirmaciones que aparecen en los anuncios. Una parte notable de los anuncios de nuestra colección en los que se invoca a la ciencia como fuente de autoridad se refieren a automóviles y a sustancias cosméticas. Las invocaciones a la ciencia como fuente de autoridad constituyen un ejemplo de una de las estrategias publicitarias habituales: el «posicionamiento». Dado que en un mercado altamente diversificado como el actual existen numerosas versiones y variedades similares de un mismo tipo de producto, la estrategia clásica para diferenciarlos en la mente del consumidor consiste en posicionarlos $\mathrm{o}$ «construirles una personalidad que les dará un lugar distinto en el mercado en relación con sus consumidores» (citado en Moliné, 1996, p. 84). Una forma de aplicar esta estrategia consiste en ofrecer al consumidor afirmaciones acerca del producto procedentes de una fuente creíble. La credibilidad de una fuente es una cualidad multidimensional en la que intervienen diversos factores (Aaker, Batra y Myers, 1992, p. 358). Algunos de estos factores, como el prestigio, la competencia o la fiabilidad, están asociados, sin duda, a las concepciones comunes sobre la ciencia, por lo que estas invocaciones genéricas a la ciencia seguramente consigan, como resultado, una mayor credibilidad para el contenido de los anuncios. De hecho, en algunos de los anuncios, los productos se presentan como solución científica a algún problema real o supuesto del consumidor (por ejemplo, «te ofrece la solución científica y eficaz»). Las invocaciones anteriores son consistentes con resultados que demuestran, pongamos por caso, que la efectividad de los anuncios comparativos es mayor cuando se invoca una fuente de gran credibilidad, como la ciencia (Gotlieb y Sarel, 1991).

En otras ocasiones, los anuncios buscan el respaldo de la ciencia destacando el carácter de vanguardia de los productos anunciados. Un estereotipo es el de avance de la ciencia. Esta idea es uno de los componentes de lo que el filósofo Stephen Brush identifica como visión whig de la ciencia, la cual es concebida como continuo avance, sin retrocesos conceptuales o marcha atrás (Brush, 1974). Según esta visión, es razonable esperar que los resultados científicos más recientes sean siempre los más valiosos, fiables y adecuados. Por otra parte, un tema común en la publicidad es «la continua alabanza de lo nuevo, que descalifica ipso facto todo lo pasado» (Brune, 1997, p. 32). De hecho, un autor clásico como Ogilvy afirma que una de las dos palabras más potentes de cualquier anuncio es nuevo (la otra es gratis) (1984, p. 142). Estas formas de neofilia en las que coinciden el mundo de la publicidad y las visiones simplistas del avance científico como un proceso de avance continuo y lineal se funden en no pocas ocasiones. Algunos anuncios insisten en que sus productos son el último resultado de la investigación científica o tecnológica. La intención más probable es transmitir a los productos el prestigio y fiabilidad que se derivaría de su condición de «productos de última vanguardia» (Meldrum, 1995, p. 47). En algún caso se afirma explícitamente que «su tecnología es tan avanzada que no se cuestiona». Estas invocaciones a la novedad como supuesto rasgo de la ciencia que garantiza la calidad de los productos son consistentes con la ausencia de anuncios que invoquen logros científicos del pasado pero todavía vigentes. Por ejemplo, seguramente no se consideraría adecuado desde el punto de vista publicitario anunciar un neumático con una afirmación como: «El comportamiento de nuestros neumáticos se rige por leyes científicas del siglo XVIII.» Sin embargo, las teorías y logros científicos que han resistido el paso del tiempo y continúan siendo utilizados actualmente deberían ser más fiables que las últimas novedades, siempre pendientes de una posterior corroboración.

\section{Usos inadecuados de los contenidos científicos en la publicidad}

Los ejemplos analizados anteriormente ilustran situaciones en las que se intenta asociar la ciencia o la tecnología a un producto determinado. Ello se hace mediante una invocación genérica a la ciencia, sin mayor profundidad o justificación. La publicidad utiliza también la ciencia presentando conceptos y principios supuestamente científicos que respaldan los productos anunciados. A continuación se presentan algunos ejemplos de esta utilización. 
Tabla II

Ejemplos de anuncios en los que aparecen conceptos inexistentes supuestamente científicos.

\begin{tabular}{|l|l|}
\hline \multicolumn{2}{|l|}{$\begin{array}{l}\text { TIPO DE } \\
\text { PRODUCTO }\end{array}$} \\
\hline $\begin{array}{l}\text { Cereales para el desayuno } \\
\text { Aceitunas }\end{array}$ & $\begin{array}{l}\text { Carbohidratos energéticamente puros, limpios. Energía sana. Baja en grasas, sin colesterol. } \\
{[\ldots] \text { mantiene su alto nivel nutritivo y biológico. }}\end{array}$ \\
$\begin{array}{l}\text { Hornos } \\
\text { Producto anticaída } \\
\text { en farmacilo de venta } \\
\begin{array}{l}\text { Crema anticelulítica } \\
\text { Crema antiarrugas }\end{array}\end{array}$ & $\begin{array}{l}\text { Una verdadera cura de energía. } \\
\text { Detergente para la ropa }\end{array}$ \\
\hline
\end{tabular}

\section{Conceptos supuestamente científicos}

Una primera fuente de ejemplos tiene que ver con el uso de conceptos supuestamente científicos. En la tabla II se recogen algunos anuncios en los que se da esta situación. Algunos de los conceptos que aparecen en la tabla son inadecuados porque se presentan ligados a adjetivos que alteran el significado original dando como resultado una combinación ininteligible (por ejemplo, acción efervescente, silicio reestructurante, calor halógeno, energía sana). En otros casos, los conceptos científicos se utilizan con una notable falta de rigor, de una manera más propia del lenguaje común e incorrecta desde el punto de vista científico. Este uso inadecuado consiste en otorgar atributos o establecer relaciones difícilmente compatibles con el significado de ciertos conceptos (por ejemplo, alto nivel biológico, cura de energía).

Los supuestos conceptos científicos que se presentan en los ejemplos deberían ser desconocidos para el lector y las frases en que aparecen deberían ser, por tanto, ininteligibles. Ello contradice la suposición de que «el consumidor espera de la publicidad información, entretenimiento y confianza» (Bassat, 1994, p. 15). En los casos que nos ocupan, los consumidores no son capaces de darse cuenta de que están leyendo un mensaje sin contenido semántico claro. Es un fenómeno similar a otro que se da frecuentemente en el aprendizaje de las ciencias: el «conocimiento ilusorio», o no darse cuenta de que no se ha entendido algo y creer al mismo tiempo que sí se ha entendido (Otero y Campanario, 1990; Campanario, 1995).

Cabe preguntarse entonces por qué se incluyen tales conceptos en los anuncios publicitarios. Una posible respuesta es que, de nuevo, se trata de transmitir un cierto carácter «científico» al discurso publicitario y de relacionar los productos que se anuncian con la ciencia o la tecnología. Seguramente se pretende conseguir el mismo efecto que con la invocación estudiada en el apartado anterior, pero recurriendo en este caso a una justificación o explicación más refinada y detallada que la mera mención genérica de la ciencia. La aparente información o justificación usando conceptos inexistentes crea la impresión de una explicación científica o de un razonamiento sólido. Probablemente los autores de los anuncios confían explícita o implícitamente encontrar un público poco dado a analizar críticamente el contenido de los mensajes científicos que recibe. Es posible que este análisis crítico, ausente, en general, del pensamiento cotidiano, constituya precisamente un ejercicio apropiado para la clase de ciencias.

\section{Errores en el uso de conceptos científicos}

En ocasiones, los conceptos científicos que aparecen en la publicidad, aun siendo correctos, se utilizan de manera inadecuada o errónea. En la tabla III se presentan ejemplos de este tipo de situaciones. Algunos de los errores podrían ser detectados, en principio, incluso por alumnos de enseñanza primaria (por ejemplo, «el aire no pesa»). Esta circunstancia plantea nuevas dudas sobre el carácter informativo de la publicidad implicada.

\section{Exageraciones}

Como señala Brune, «a través de su diversidad aparente, todas las publicidades celebran el producto como héroe» (Brune, 1997, p. 32), y quizá la forma más característica de alabanza sea exagerar sus cualidades. La hipérbole es una de las figuras retóricas clásicas y también es uno de los recursos publicitarios para mitificar el producto 
Tabla III

Ejemplos de anuncios en los que se utilizan conceptos científicos de manera inadecuada o aparecen afirmaciones incorrectas desde el punto de vista científico.

\begin{tabular}{|c|c|}
\hline $\begin{array}{l}\text { TIPO DE } \\
\text { PRODUCTO }\end{array}$ & CONTENIDO DEL ANUNCIO \\
\hline Crema hidratante & $\begin{array}{l}\text { «Con [...] el agua fisiológica es canalizada y dominada, proporcionando así a su piel recursos permanentes de } \\
\text { hidratación.» }\end{array}$ \\
\hline Crema hidratante & $\begin{array}{l}\text { «El magnesio, fuente de energía.» } \\
\text { El magnesio, por sí mismo, no es una fuente de energía. Es posible que el magnesio o algún compuesto en el } \\
\text { que intervenga el magnesio pueda dar lugar a reacciones bioquímicas en las que se desprenda energía, pero } \\
\text { esto es también cierto probablemente para muchos otros elementos químicos. }\end{array}$ \\
\hline $\begin{array}{l}\text { Cámara fotográfica } \\
\text { digital }\end{array}$ & $\begin{array}{l}\text { «Porque la [...] proporciona una sorprendente resolución de } 570.000 \text { pixels.» } \\
\text { El número de pixels está relacionado con la resolución, pero, sin conocer la superficie real que cubren estos } \\
\text { pixels, no se puede tener una idea exacta de la resolución de las imágenes. }\end{array}$ \\
\hline Reloj & $\begin{array}{l}\text { «[...] su robusta caja, que se esculpe en un bloque macizo de metal, utilizando hasta } 60 \text { toneladas de presión.» } \\
\text { La presión no se mide en toneladas (unidad de medida de masa). Admitamos que se utiliza esta unidad de masa } \\
\text { para transmitir una idea de la fuerza que se utiliza. No obstante, si no se conoce la superficie sobre la que se } \\
\text { aplica la fuerza correspondiente, no podemos saber cómo es de grande la presión. }\end{array}$ \\
\hline Hornos & $\begin{array}{l}\text { «La puerta fría evita el riesgo de quemaduras gracias a su cristal interior, que impide que las altas temperaturas } \\
\text { salgan al exterior.» } \\
\text { Una forma posible de evitar que se transfiera el calor al exterior es utilizar una puerta aislante, no una puerta } \\
\text { fría. Ese calor que se transfiere al exterior es, en todo caso, el responsable del aumento de la temperatura en } \\
\text { el exterior. La temperatura, por sí misma, no «sale». }\end{array}$ \\
\hline Azúcar & «El azúcar es la fuerza de la naturaleza.» \\
\hline Cosmético & $\begin{array}{l}\text { «Su buen funcionamiento depende de la presencia en el medio extracelular de unas moléculas inteligentes } \\
\text { llamadas citoquinonas.» } \\
\text { Este ejemplo ilustra el abuso de las palabras inteligente e inteligencia que, últimamente, se atribuye a casi todo, } \\
\text { desde refrescos hasta automóviles. }\end{array}$ \\
\hline Automóvil & $\begin{array}{l}\text { «Su sofisticado sistema [...], con su avanzada capacidad de autodiagnóstico, es capaz de pensar } 231.800 \text { veces } \\
\text { por segundo.» } \\
\text { Más fácil todavía, ¿qué significaría, por ejemplo, pensar } 3 \text { veces por segundo? }\end{array}$ \\
\hline Helados & $\begin{array}{l}\text { «Todos sus ingredientes principales son naturales: leche, azúcar, yema de huevo [...]» } \\
\text { El azúcar que consumimos es un producto que requiere elaboración. Seguramente los ingredientes principales } \\
\text { de casi todos los alimentos, incluso los elaborados, también sean naturales. Además, el que los productos sean } \\
\text { naturales no garantiza que no sean perjudiciales en cualquier cantidad o circunstancia, dado que muchos } \\
\text { productos naturales son nocivos. }\end{array}$ \\
\hline Crema hidratante & $\begin{array}{l}\text { «Las } 2 / 3 \text { partes de nuestra piel podrían evaporarse. Con }[\ldots] \text { el agua se fija en la piel de forma duradera.» } \\
\text { Tal vez se refiera al agua de la piel que, efectivamente, fuera de las células puede evaporarse. De la misma } \\
\text { manera se puede afirmar que un porcentaje elevado del cuerpo humano podría evaporarse. }\end{array}$ \\
\hline Helados & «Porque el aire no pesa.» \\
\hline Crema & $\begin{array}{l}\text { «Energía regeneradora para el tratamiento de las arrugas.» } \\
\text { Utilización inadecuada de la palabra energía. }\end{array}$ \\
\hline Colirio & $\begin{array}{l}\text { «El principio activo de [...] es agua destilada de la flor de hamamelis.» } \\
\text { El agua destilada sólo es agua. El principio activo debería contener algo más. }\end{array}$ \\
\hline
\end{tabular}

(McQuarrie y Mick, 1996). En la tabla IV se recogen ejemplos de hipérboles o de exageraciones que dan lugar a errores y falsedades. Como es de esperar, estas exageraciones están asociadas al uso de calificativos y expresiones excluyentes, absolutas o superlativas (por ejemplo, máximo, total, imposible, en todo momento...). De confiar en la función informativa de la publicidad, algunos usuarios de ciertos productos que se anuncian de esta forma verían peligrar su integridad física. Ejemplos de ello son los anuncios que se refieren a una "carrocería indeformable»o a un neumático «que evita cualquier deslizamiento».

La exageración, los errores y las promesas sobre virtudes del producto imposibles de cumplir que se aprecian en los anuncios de la tabla IV contrastan con una recomendación clásica de la teoría de la publicidad que, en palabras de Bassat se formula así: [...] la publicidad no 
Tabla IV

Ejemplos de anuncios en los que aparecen hipérboles o se formulan afirmaciones claramente exageradas que son incorrectas.

\begin{tabular}{|c|c|}
\hline $\begin{array}{l}\text { TIPO DE } \\
\text { PRODUCTO }\end{array}$ & CONTENIDO DEL ANUNCIO \\
\hline Automóvil & $\begin{array}{l}\text { «Es imposible copiar o descubrir la clave.» } \\
\text { No existe todavía ningún algoritmo de codificación completamente invulnerable. }\end{array}$ \\
\hline Automóvil & $\begin{array}{l}\text { «Un control absoluto de cada situación, por muy difícil que ésta sea.» } \\
\text { No parece probable que exista el control absoluto de cualquier situación. }\end{array}$ \\
\hline Automóvil & $\begin{array}{l}\text { «El sistema antirrobo cuenta con una infinita combinación de códigos.» } \\
\text { El anuncio está mal redactado: debería decir infinitas combinaciones de códigos. En cualquier caso es } \\
\text { imposible conseguir un número infinito de combinaciones, tal como se entiende en el análisis combinatorio, a } \\
\text { partir de un número reducido de elementos. }\end{array}$ \\
\hline Automóvil & $\begin{array}{l}\text { «Sus neumáticos de perfil bajo le garantizan una adherencia total en todo tipo de terreno.» } \\
\text { No es posible la adherencia total, mucho menos en todo tipo de terreno. }\end{array}$ \\
\hline Neumáticos & $\begin{array}{l}\text { «Un neumático que evita cualquier deslizamiento, haya lo que haya entre la carretera y su coche.» } \\
\text { Esta exageración es también errónea y peligrosa. Para evitar cualquier deslizamiento se necesitaría un } \\
\text { coeficiente de rozamiento entre las ruedas y el suelo que fuese infinito. Sin embargo, admitamos, en principio, } \\
\text { que el coeficiente de rozamiento entre las ruedas y el suelo sea muy grande. Ello dificulta los deslizamientos, } \\
\text { aunque no puede impedirlos totalmente y en cualquier circunstancia. Si se permite que exista algo entre la } \\
\text { rueda y el suelo, como un lubricante, el coeficiente de rozamiento puede disminuir considerablemente, con lo } \\
\text { cual es posible que existan deslizamientos incluso a velocidades muy pequeñas. }\end{array}$ \\
\hline Diamantes & $\begin{array}{l}\text { «e formó hace tres mil millones de años (aproximadamente), casi antes del principio de los tiempos y, por } \\
\text { supuesto, mucho antes de la Creación.» } \\
\text { La antigüedad que se atribuye al diamante no es superior a la de otras rocas y minerales muy comunes sobre } \\
\text { la superficie terrestre. Por otra parte, tres mil millones de años no es, como afirma el anuncio, casi antes del } \\
\text { principio de los tiempos. La edad del universo se estima en veinte mil millones de años y la de la Tierra en unos } \\
\text { cuatro mil quinientos millones de años. Además, existe una contradicción interna entre la frase «casi antes del } \\
\text { principio de los tiempos»y la frase «mucho antes de la creación». }\end{array}$ \\
\hline Automóvil & «Tacaño porque apenas mueve el indicador del depósito por larga que sea la distancia.» \\
\hline Automóvil & $\begin{array}{l}\ll[\ldots] \text { un todoterreno que puede con todo.» } \\
\text { Sin importar el terreno en el que se mueva ni la cantidad de equipaje que tenga que transportar. }\end{array}$ \\
\hline Automóvil & $\begin{array}{l}\text { «Su carrocería indeformable.» } \\
\text { ¿Es realmente indeformable toda la carrocería, aun bajo el efecto de cualquier fuerza? Además, una carrocería } \\
\text { indeformable implica que no se realizaría ningún trabajo para deformarla en una colisión, con el consiguiente } \\
\text { peligro para los pasajeros. }\end{array}$ \\
\hline Lentes & «Resisten todo tipo de choques.» \\
\hline Colchón & «Hasta dos mil muelles entrelazados y formados por un solo hilo. Indeformables. Indestructibles.» \\
\hline
\end{tabular}

debe defraudar. Y no me refiero a defraudar las expectativas creadas con el producto, aunque también sea este uno de los pecados más graves que se cometen, sino de algo incluso anterior en el tiempo. Hablo de no defraudar al consumidor preocupado por el riesgo que debe tomar, ansioso de encontrar información y argumentos contundentes en nuestras comunicaciones» (Bassat, 1994; p. 16).

\section{Razonamientos incorrectos}

En un estudio realizado en 1984, consultamos a un grupo reducido de científicos sobre los objetivos de la enseñanza de las ciencias en España como parte de la educa- ción general (no como preparación para futuros estudios científicos o técnicos). El grupo de expertos generó un conjunto de 85 objetivos para los niveles de enseñanza primaria y bachillerato. En una segunda fase se les solicitó la valoración de los objetivos en una escala de 1 a 5 . Hubo acuerdo de todos los científicos en otorgar la máxima valoración a 3 de los objetivos. Dos de éstos eran los siguientes:

«El ciudadano educado de la década de los noventa:

- será capaz de desarrollar una argumentación lógica;

- será capaz de distinguir entre impresiones, suposiciones o interpretaciones, y observaciones o verdades de- 
mostradas experimentalmente» (Otero, Aguirre de Cárcer, Bourgeal y Conde, 1984, p. 87).

Este resultado pone de manifiesto la importancia que los científicos preguntados otorgaban al desarrollo de modos de razonamiento asociados al pensamiento científico, como parte del bagaje cultural de cualquier ciudadano. Éste se considera uno de los resultados más valiosos de la interacción de los ciudadanos con las ciencias durante su escolarización. Los estudiantes deben aprender a desarrollar y distinguir argumentaciones lógicas y convertirse «en pensadores independientes, capaces de adquirir información procedente de varias fuentes, de ponderar alternativas y de alcanzar conclusiones defendibles» (Watson, 1983, p. 62). La publicidad, sin embargo, utiliza, en ocasiones como las recogidas en los ejemplos de las tablas V y VI, argumentaciones inconsistentes, deducciones erróneas o comparaciones incompletas; en suma, razonamiento de muy baja calidad.

En la tabla V aparecen ejemplos de comparaciones cuantitativas incompletas. Es evidente que en toda comparación se necesitan al menos dos elementos y una medida de la semejanza o diferencia entre ellos. En algunos de los ejemplos de la tabla V, o falta uno de los elementos de la comparación, o falta el grado que diferencia los elementos que se comparan, o faltan ambas cosas. El uso de expresiones estereotipadas (por ejemplo, más sabor) ha terminado por vaciarlas de contenido, de tal manera que ya casi nadie parece reparar en la falta de elementos de comparación. Más llamativo es que en algunos de los ejemplos de la tabla $\mathrm{V}$ aparezca una

Tabla V

Ejemplos de anuncios que presentan comparaciones cuantitativas inadecuadas, incompletas, indeterminadas o unilaterales

\begin{tabular}{|c|c|}
\hline $\begin{array}{l}\text { TIPO DE } \\
\text { PRODUCTO }\end{array}$ & CONTENIDO DEL ANUNCIO \\
\hline Automóvil & «Tecnología más segura para el medio ambiente.» \\
\hline $\begin{array}{l}\text { Vehículo } \\
\text { industrial } \\
\text { (furgoneta) }\end{array}$ & $\begin{array}{l}\text { «Un } 10 \% \text { más de potencia. Un } 17 \% \text { menos de consumo.» } \\
\text { En los dos casos anteriores no se cita ningún vehículo con el cual se compare el vehículo que se anuncia. }\end{array}$ \\
\hline Bombilla & $\begin{array}{l}\ll[\ldots] \text { producimos bombillas que duran diez veces más y consumen un } 80 \% \text { menos de energía.» } \\
\text { Tampoco se cita ninguna bombilla con la cual comparar. }\end{array}$ \\
\hline $\begin{array}{l}\text { Vehículos } \\
\text { industriales } \\
\text { (camiones) }\end{array}$ & $\begin{array}{l}\text { «Un }[. . .] \text { de cuarenta toneladas tarda menos en frenar que usted en leer esto.» } \\
\text { La afirmación anterior parece implicar que el camión emplea muy poco tiempo en frenar. Seguramente el lector } \\
\text { supone que el camión frena desde una velocidad inicial normal o alta. Sin embargo, dado que no se indica ni } \\
\text { la aceleración ni la velocidad inicial del camión cuando empieza a frenar, el anunciante no se compromete a } \\
\text { mucho. }\end{array}$ \\
\hline Automóvil & $\begin{array}{l}\text { «En lo que tarda en caer una gota de lluvia, un [...] toma más de doscientas decisiones para poder esquivarla.» } \\
\text { El problema con este anuncio es similar al que existe con el anuncio anterior. La afirmación parece querer } \\
\text { indicar que el automóvil «toma» muchas decisiones en poco tiempo. Sin embargo, dado que no se indica desde } \\
\text { qué altura cae la gota de agua, el tiempo que transcurre en dicha caída queda indeterminado. Además, puede } \\
\text { resultar sumamente difícil que un coche evite una gota de lluvia. Para ello primero necesitaría disponer de un } \\
\text { sistema para detectarla y, a continuación, debería alterar la trayectoria o la velocidad, algo que no siempre } \\
\text { es posible en una carretera. En todo caso, el evitar la gota de lluvia depende de la trayectoria de la gota, de } \\
\text { la velocidad de caída de la misma, de la trayectoria del coche y de su velocidad. Por último, ¿que ventajas tiene } \\
\text { para un coche evitar una gota de lluvia? }\end{array}$ \\
\hline Helados & $\begin{array}{l}\text { «[...] elabora sus helados con el doble de calidad.» } \\
\text { No se cita otro producto con el que comparar. Además, resulta difícil cuantificar la calidad. }\end{array}$ \\
\hline Bombilla & $\begin{array}{l}\text { «100W }-20 \mathrm{~W}=80 \mathrm{~W} \quad 80 \mathrm{~W} * 14 \text { pts./Kwh } * 4.000 \text { horas/año }=4.800 \text { pts. de ahorro anual. } \\
\text { En efecto, sustituyendo una bombilla tradicional de } 100 \mathrm{~W} \text { luciendo } 12 \text { horas diarias, por una [...] de } 20 \mathrm{~W} \text {, se } \\
\text { ahorra, a lo largo de dos años hasta } 10.000 \text { pts.; unas tres veces el valor de su inversión.» } \\
\text { La comparación se basa en suponer que una bombilla está luciendo } 12 \text { horas diarias, } 365 \text { días al año. Una } \\
\text { situación poco probable. Además } 12 \text { horas diarias durante } 365 \text { días al año son } 4.380 \text { horas, más de lo que se } \\
\text { indica arriba. El cálculo de arriba es incorrecto: } 0,080 \mathrm{Kw} * 14 \text { pts./Kwh } * 4.000 \mathrm{~h} / \text { año }=4.480 \text { pts./año. Incluso } \\
\text { un cálculo basado en los datos de la segunda comparación es inexacto: } 0,080 \mathrm{Kw} * 14 \text { pts./Kwh } * 12 \mathrm{~h} / \text { día } * 365 \\
\text { días } / \text { año }=4.906 \text { pts. } / \text { año } * 2 \text { años }=9.812 \text { pts. y no } 10.000 \text { pts. }\end{array}$ \\
\hline Crema & $\begin{array}{l}\text { «[..] gracias al aceite de }[\ldots] \text { que les proporciona una acción revitalizante y antienvejecimiento } 4 \text { veces más } \\
\text { importante.» } \\
\text { No se compara con ningún otro producto. No se indica cómo se puede medir cuantitativamente la importancia. }\end{array}$ \\
\hline
\end{tabular}


Ejemplos de anuncios que presentan razonamientos o argumentaciones falaces.

\begin{tabular}{|c|c|}
\hline $\begin{array}{l}\text { TIPO DE } \\
\text { PRODUCTO }\end{array}$ & CONTENIDO DEL ANUNCIO \\
\hline $\begin{array}{l}\text { Pastillas para facilitar } \\
\text { la digestión }\end{array}$ & $\begin{array}{l}\text { «Contiene [...] y dimeticona, que rompe las burbujas de aire, eliminando los gases.» } \\
\text { Romper las burbujas implica sólo que desaparece la capa líquida que envuelve los gases, pero éstos seguirán } \\
\text { allí y se difundirán por el espacio cercano. }\end{array}$ \\
\hline Automóvil & $\begin{array}{l}\ll[\ldots] \text { gracias a su ordenador de gestión integral }[\ldots] \text { capaz de procesar } 1.200 .000 \text { datos por segundo.» } \\
\text { Hay muchas formas de procesar datos. ¿Que debemos entender aquí por procesar? }\end{array}$ \\
\hline Aceite hidratante & $\begin{array}{l}\text { «Aloe vera, la planta del desierto, capaz de vivir en condiciones extremas gracias a sus propiedades para retener } \\
\text { la humedad, y que, utilizada en belleza, ayuda a mantener la humedad natural de la piel.» } \\
\text { Las características fisiológicas de una planta que le permiten vivir en condiciones extremas probablemente } \\
\text { tengan poco que ver con las propiedades bioquímicas y su actuación sobre los tejidos cutáneos. }\end{array}$ \\
\hline Cosmético & $\begin{array}{l}\text { «Su fórmula de un riqueza extrema ( } 94,8 \% \text { de principios activos) corrige las imperfecciones de la fibra capilar.» } \\
\text { La concentración de «principios activos» no es garantía de una mayor efectividad. En determinadas situaciones } \\
\text { puede resultar conveniente utilizar disoluciones más diluidas, por lo que la elevada concentración no garantiza } \\
\text { nada. }\end{array}$ \\
\hline
\end{tabular}

diferencia cuantitativa, faltando uno de los elementos de comparación (por ejemplo, un $10 \%$ más de potencia) sin que aparezca el término de referencia. Este tipo de comparaciones carece, evidentemente, de significado.

Especialmente interesantes son los ejemplos de la tabla $\mathrm{V}$, en que aparecen comparaciones que se refieren al tiempo de frenado de un camión y al tiempo de caída de una gota de agua. El objetivo de las frases implicadas parece ser llamar la atención de un lector que, en principio, no está obligado a leer el resto del anuncio (McQuarrie y Mick, 1996). Ésta puede ser la razón por la que se recurre a afirmaciones tan aparentemente rotundas pero que, como se explica en la tabla $\mathrm{V}$, de poco informan, dado que las comparaciones son indeterminadas. Se precisa un análisis con cierto detalle para descubrir la indeterminación. Sin embargo, los enunciados de los dos anuncios tienen una aparente y engañosa sencillez. Estos ejemplos ilustran un uso inteligente de la ambigüedad, de manera que, al igual que en cualquier proceso de comprensión de textos, es el propio lector el que activa un esquema que permite rellenar por defecto los huecos en la información externa (Rumelhart, 1980). El esquema permite completar determinada información que es necesaria para entender el anuncio, tal como desea el publicista, pero sin que éste se vea comprometido a respaldar inferencias generadas solamente por la persona que lee el anuncio. Así, por ejemplo, en el anuncio que se refiere al tiempo de frenado de un camión, el lector activa probablemente un esquema relativo a frenos muy eficaces, sin que las afirmaciones del anuncio garanticen esa suposición, dado que son compatibles con varias formas de frenado. Ejemplos similares de anuncios, en los cuales se espera que el lector aporte una parte importante de la información necesaria para entenderlos, han sido estudiados por van Eemeren, Grootendorst, Jack- son y Jacobs (1997). Estos autores han analizado las argumentaciones que aparecen en dos anuncios sobre publicidad del tabaco y demuestran que, cuando el lector aporta la información necesaria, de la lectura del anuncio se desprende un mensaje real contrario al que aparentemente se transmite (en este caso, que la decisión de fumar debería corresponder sólo a personas adultas).

En los ejemplos que aparecen en la tabla VI, hay anuncios que presentan razonamientos o argumentaciones falaces. En algunos casos no parece existir relación entre las supuestas cualidades de los productos que se anuncian y los efectos que producen (por ejemplo, ¿en qué se basa la relación entre la resistencia de una planta a la sequía y la capacidad de sus componentes para mantener la humedad de la piel?). En otro ejemplo, el proceso de eliminación de burbujas no explica qué le sucede al gas contenido en la burbuja. A pesar de la falta de fundamentación de la argumentación, las expresiones que se recogen son, de nuevo, de gran sencillez aparente, lo cual es consistente con uno de los postulados básicos de la publicidad: «en la buena publicidad, la idea es simple, clara y se entiende a la primera [...]. El ingenio creativo no se demuestra con analogías complicadas o historias rebuscadas» (Bassat, 1994, p. 85). De nuevo, en estos ejemplos parece que predomina el parecer sobre el ser.

El caso de los ejemplos recogidos en la tabla VII es diferente. En estos ejemplos aparecen explicaciones y razonamientos que parecen excesivamente sofisticados y difíciles de entender, al menos para un ciudadano medio. Parte de las dificultades se deben a los conceptos técnicos y científicos que se citan en los anuncios y a la complejidad de los procesos implicados. Mientras que en los ejemplos de las tablas V y VI se hace creer al consumidor que está ante un razonamiento comprensi- 
TablaVII

Ejemplos de anuncios en los que se presentan argumentaciones sofisticadas y difíciles de entender.

\begin{tabular}{|c|c|}
\hline $\begin{array}{l}\text { TIPO DE } \\
\text { PRODUCTO }\end{array}$ & CONTENIDO DEL ANUNCIO \\
\hline Automóvil & $\begin{array}{l}\text { «a suspensión [...] es independiente en las cuatro ruedas, con tren delantero triangulado de geometría } \\
\text { optimizada y tren trasero de doble triangulación superpuesta y planos controlados con gestión electrónica } \\
\text { de amortiguación.» }\end{array}$ \\
\hline Automóvil & $\begin{array}{l}\text { «La suspensión delantera y trasera monta muelles helicoidales de doble efecto, siendo las traseras de endure } \\
\text { cimiento progresivo de acuerdo con los esfuerzos requeridos.» }\end{array}$ \\
\hline Automóvil & $\begin{array}{l}\text { «Tren delantero de geometría perfeccionada que hace que las ruedas se beneficien siempre del ángulo de } \\
\text { ataque adecuado en relación con la carretera.» }\end{array}$ \\
\hline Bujía & «La nueva bujía $[\ldots]$ que trabaja según el principio de chispa deslizante en el aire.» \\
\hline Aceite corporal & «La innovadora emulsión $W$-O-W (agua en aceite en agua).» \\
\hline
\end{tabular}

ble (aunque, como se ha indicado, no es así), en los ejemplos de la tabla VII parece que se desea justamente lo contrario: situar al consumidor ante la evidencia de que la complejidad técnica está más allá de sus posibilidades de comprensión. De nuevo, la intención de estos anuncios no parece que sea informar a los consumidores para que comprendan las características de lo que se anuncia. El propósito parece, más bien, informar de la complejidad del producto o de su funcionamiento, de una manera indirecta. En lugar de explicar cuál es la función de la «doble triangulación superpuesta» de un tren trasero, el publicista transmite la idea de que el dispositivo es de gran complejidad, mediante una descripción que sea ininteligible para el destinatario del mensaje. Seguramente se espera que el lector infiera el mensaje final: no se entiende el funcionamiento, luego debe ser complejo; si es complejo, es valioso.

El análisis de los anuncios de este apartado sugiere que los responsables del diseño publicitario mantienen ciertas suposiciones sobre el destinatario de sus mensajes: o bien consideran que no está dotado de las destrezas intelectuales necesarias para analizar la argumentación que se presenta e identificar las inconsistencias existentes, o quizá consideren que, aun teniendo esta capacidad, no la pondrá en práctica.

\section{CONCLUSIONES}

En los últimos años se ha agudizado el debate en torno a la publicidad. La sobrecarga publicitaria que inunda al ciudadano desde todos los canales y medios informativos es tal que la eficacia de la misma es cada vez menor (Romeu, 1996). Algunos autores hablan abiertamente de la existencia de una crisis en la publicidad (Costa, 1995). En una situación de saturación informativa y publicitaria es cada vez más difícil que los consumidores presten su atención a un reclamo determinado o diferencien un producto del maremagnum de propuestas que se les ofrece. No es sorprendente que la ciencia o el lenguaje científico se cuenten entre los recursos que se utilizan para llamar la atención de los consumidores y para aumentar la credibilidad de los anuncios.

Los ejemplos seleccionados muestran, en primer lugar, que la ciencia se utiliza en la publicidad como fuente de autoridad que respalda las virtudes de los productos anunciados. Existen formas diversas de invocar la ciencia como fuente de garantía, calidad o eficacia. Estas formas van desde la mera asociación de un producto a la ciencia o a la tecnología hasta la afirmación explícita de que la ciencia respalda la calidad del producto. El uso de la autoridad de la ciencia, y de la concepción asociada de conocimiento científico como conocimiento verdadero, es posible que no se limite a aprovechar unos esquemas mentales preexistentes sino que además contribuye a reforzar en el público esta imagen errónea de la ciencia. En este sentido la publicidad enseña lecciones inconsistentes con las pertenecientes a la ciencia escolar.

En segundo lugar, los análisis anteriores muestran que el contenido de muchos anuncios es deficiente desde el punto de vista científico. Los ejemplos seleccionados incluyen falsedades, conceptos supuestamente científicos, razonamientos falaces, comparaciones cuantitativas incompletas o unilaterales y afirmaciones deliberadamente incomprensibles. En todas estas situaciones, las violaciones al uso del lenguaje científico y a la lógica hacen que el contenido informativo del anuncio sea mínimo o nulo. Se trata de un uso de la ciencia fundamentado en las impresiones superficiales, más que en la comprensión. Sin embargo, el uso inadecuado que se hace de estos recursos debe ser eficaz y rentable para los anunciantes. Ello implica una cierta concepción de los conocimientos y destrezas intelectuales del destinatario: los publicistas deben contar con la insensibilidad de los 
consumidores a las inconsistencias e incorrecciones en sus mensajes. Por ello, el uso inadecuado de la ciencia en la publicidad plantea dudas sobre el nivel de conocimientos científicos y destrezas de razonamiento de las personas a quienes se destinan estos anuncios. Sin embargo, más que culpar a la publicidad de utilizar inadecuadamente la ciencia, cabría culpar a los consumidores por otorgar su confianza, a través de su poder de compra, a tales prácticas. Al igual que la publicidad aprovecha y utiliza para el logro de sus objetivos determinados gustos y costumbres sociales, la mala formación científica de los ciudadanos permite y favorece usos inadecuados de la ciencia como los que se ilustran en los ejemplos que hemos discutido.

Es preciso reconocer que no es fácil explicar de manera clara en un breve anuncio, a un auditorio heterogéneo, los mecanismos científicos o técnicos de actuación de un producto y las cualidades que lo hacen bueno o que garantizan su eficacia. La comprensión de la actuación de un cosmético, por ejemplo, requiere conocimientos de bioquímica. Es, por tanto, más fácil invocar a la ciencia o a la investigación de manera genérica, o crear la impresión de que se ha presentado una argumentación científica y rigurosa cuando en realidad no es así. Ello es coherente con algunas orientaciones clásicas del marketing. Así, por ejemplo, Trout y Ries, autores de un best-séller en el área publicitaria, afirman abiertamente que «el marketing no es una batalla de productos, es una batalla de percepciones. La mayoría de los errores de marketing nacen del supuesto de que se libra una batalla de productos enraizada en la realidad. No hay mejores productos. En marketing, lo único que existe son percepciones en la mente de los clientes actuales y potenciales. La percepción es la realidad, todo lo demás es ilusión» (citado por Peralba y González, 1996, p. 40). El problema está en que, de nuevo, estos procedimientos transmiten lecciones contradictorias con los objetivos que el sistema educativo persigue con la enseñanza de las ciencias. Así, mientras los profesores intentan que los alumnos comprendan, analicen y apliquen de manera reflexiva los conocimientos científicos, huyan del aprendizaje memorístico y lleguen a la convicción de que la ciencia es una empresa basada en la racionalidad, con reglas y procedimientos inteligibles, cierta publicidad transmite la lección opuesta. Para transmitir la idea de que un producto es valioso, en algunos de los ejemplos estudiados más arriba, el publicista presenta una imagen compleja de sus componentes, o del proceso de fabricación, a través de una descripción científica o técnica incomprensible. Se espera que los sujetos consideren normal no entender un mensaje científico ( aun cuando está dirigido al público en general!), dada la complejidad de la ciencia, en lugar de criticar la torpeza del que lo ha producido.

El hecho de que los ciudadanos sean aparentemente insensibles a las exageraciones en la publicidad, al uso de conceptos científicos inexistentes, o a las argumentaciones falaces o incomprensibles debería llevar a cuestionar los logros reales de la enseñanza de las ciencias y su efecto sobre el nivel cultural medio de la población: la llamada alfabetización científica. Además, no es la publicidad el único contexto en el que parecen ser aceptables las argumentaciones inadecuadas o incomprensibles. El discurso político es también propicio a este tipo de abusos (Otero, 1996). Por tanto, presentamos el análisis que hemos llevado a cabo como un elemento a tener en cuenta en los fines, los métodos y los resultados de la educación científica y crítica de los ciudadanos; en suma, en la consideración permanente de la función de la educación general en una sociedad compleja y cambiante en la que la ciencia y la tecnología juegan un papel destacado.

\section{AGRADECIMIENTOS}

Agradecemos los comentarios y sugerencias de los asesores de la revista Enseñanza de las Ciencias.

\section{REFERENCIAS BIBLIOGRÁFICAS}

AAKER, D.A., BARTRA, R. y MYERS, J.G. (1992). Advertising Management. Englewood Cliffs, N.J.: Prentice-Hall International.

BASSAT, L. (1994). El libro rojo de la publicidad. Barcelona: Folio.

BRUNE, F. (1997). Agresiones publicitarias. Le Monde Diplomatique, 20 , p. 32.
BRUSH, S.G. (1974). Should the history of science be rated X? Science, 183, pp. 1164-1172.

CAMPANARIO, J.M. (1995). Los problemas crecen: a veces los alumnos no se enteran de que no se enteran. Aspectos didácticos de Física y Química (Física) 6, pp. 87-126. Zaragoza: ICE, Universidad de Zaragoza.

COOK, G. (1992). The Discourse of Advertising. Londres: Routledge. 
COSTA, J. (1995). Crisis y crítica de la publicidad. Un narciso que se cree Jano. Telos, 42, pp. 28-34.

ELTINGE, E.M. y ROBERTS, C.W. (1993). Linguistic content analysis: A method to measure science as inquiry in textbooks. Journal of Research in Science Teaching, 30, pp. 65-83.

GALBRAITH, J.K. (1992). La sociedad opulenta. Barcelona: Ariel.

GOTLIEB, J.B. y SAREL, D. (1991). Comparative advertising effectiveness: The role of involvement and source credibility. Journal of Advertising, 20, pp. 38-44.

INFOADEX (1997). Inversión 96: 1.251 .962 pesetas. Ipmark, 487, pp. $12-14$

LAKATOS, I. y MUSGRAVE, A. (1975). La crítica y el desarrollo del conocimiento. Barcelona: Grijalbo.

LEDERMAN, N.G. (1992). Students' and teachers' conceptions of the nature of science: A review of the research. Journal of Research in Science Teaching, 24, pp. 331-359.

MAcILWAIN, C. (1998). US public puts faith in science, but still lacks understanding. Nature, 394, p. 107.

McQUARRIE, E.F. y MICK, D.G. (1996). Figures of rhetoric in adverstising language. Journal of Consumer Research, 22, pp. 424-438.

MANRIQUE DEL CAMPO, M.J. (1995). Noticias para plantear problemas. Alambique, 5, pp. 59-65.

MEICHTRY, Y.J. (1993). The impact of science curricula on student views about the nature of science. Journal of Research in Science Teaching, 30, pp. 429-443.

MELDRUM, M.J. (1995). Marketing high-tech products: the emerging themes. European Journal of Marketing, 29, pp. 45-58.

MOLINÉ, M. (1996). La comunicación activa. Publicidad sólida. Bilbao: Ediciones Deusto.

MORGAN, F.W. y STOLTMAN, J.J. (1997). Advertising and product liability litigation. The Journal of Advertising, 26, pp. 63-75.

NELKIN, D. (1991). Las imágenes de la ciencia en la prensa americana. Arbor, 140, pp. 129-141.

NELKIN, D. (1990). La ciencia en el escaparate. Madrid: Fundesco.

OGILVY, D. (1984). Confesiones de un publicitario. Barcelona: Orbis.
OTERO, J.C. (1987). Comprehension monitoring in learning from scientific text. Comunicación presentada en el Second International Seminar on Students' Misconceptions and Educational Strategies in Science and Mathematics. Ithaca, Nueva York: Cornell University.

OTERO, J.C. (1996). Aportacións inesperadas o curriculum de ciencias: Contribucións dum publicista e dous líderes políticos á formación de modos de pensamento. Boletín das Ciencias, 26, pp. 61-69.

OTERO, J.C., AGUIRRE DE CÁRCER, I., BOURGEAL, S. y CONDE, L. (1984). Aplicación del método Delphi para la generación de objetivos de la formación científica y tecnológica en el nivel de la educación general, en Aguirre de Cárcer, I. (ed.). La selectividad a debate. Madrid: Servicio de Publicaciones de la UAM.

OTERO, J.C. y CAMPANARIO, J.M. (1990). Comprehension evaluation and regulation in learning from science texts. Journal of Research in Science Teaching, 27, pp. 447-460.

PERALBA, R. y GONZÁLEZ, R. (1996). Las 22 leyes inmutables del marketing (1). Ipmark, 462, pp. 38-43.

PORLÁN, R. (1994). Las concepciones epistemológicas de los profesores: el caso de los estudiantes de magisterio. Investigación en la Escuela, 22, pp. 67-84.

RAYMOND, N. (1988). Cosmetics Advertising: A Look at the Foundations. Documento CS506326, Base de Datos ERIC.

ROMEU, J. (1996). La eficacia de la publicidad ha descendido durante los últimos años. Ipmark, 468, pp. 46-47.

RUMELHART, D.E. (1980). Schemata: The building blocks of cognition, en Spiro, R.J., Bruce, B.C. y Brewer, W.F. (eds.). Theoretical issues in reading comprehension. Hillsdale, Nueva Jersey: Lawrence Erlbaum.

STAHL, W.A. (1995). Venerating the black box: Magic in media discurse on technology. Science, Technology and Human Values, 20, pp. 234-258.

VAN EEMEREN, F.H., GROOTENDORST, R., JACKSON, S. y JACOBS, S. (1997). Argumentation, en van Dijk, T.A. (ed.). Discourse as Structure and Process. Londres: Sage.

WATSON, F. (1983). On the drawing board: A 21st century curriculum. The Science Teacher, 50, pp. 62-63.

YEARLEY, S. (1993-1994). La autoridad social de la ciencia en la edad postmoderna. Política y Sociedad, 14-15, pp. $59-66$

[Artículo recibido en diciembre de 1997 y aceptado en diciembre de 1998.] 